PROCEEDINGS OF THE

AMERICAN MATHEMATICAL SOCIETY

Volume 138, Number 11, November 2010, Pages 3797-3805

S 0002-9939(2010)10405-7

Article electronically published on May 18, 2010

\title{
LINEAR SERIES ON RIBBONS
}

\author{
DAWEI CHEN
}

(Communicated by Ted Chinburg)

\begin{abstract}
A ribbon is a double structure on $\mathbb{P}^{1}$. The geometry of a ribbon is closely related to that of a smooth curve. In this paper we consider linear series on ribbons. Our main result is an explicit determinantal description for the locus $W_{2 n}^{r}$ of degree $2 n$ line bundles with at least $(r+1)$-dimensional sections on a ribbon. We also discuss some results of Clifford and Brill-Noether type.
\end{abstract}

\section{INTRODUCTION}

In this section, we recall some basic theory of ribbons. In the literature a ribbon is also called a Fossum-Ferrand doubling structure. Here we will mainly follow Bayer-Eisenbud [BE] for the related terminology. Many results and much of the notation below come from their paper.

We work over an algebraically closed field $k$ of characteristic 0 . A ribbon on $\mathbb{P}^{1}$ is a scheme $C$ equipped with an isomorphism $\mathbb{P}^{1} \rightarrow C_{r e d}$, such that the ideal sheaf $\mathscr{L}$ of $\mathbb{P}^{1}$ in $C$ satisfies

$$
\mathscr{L}^{2}=0
$$

Because of this condition, $\mathscr{L}$ can be regarded as a line bundle on $\mathbb{P}^{1}$. It is called the conormal bundle of $\mathbb{P}^{1}$ in $C$. There is a short exact sequence called the conormal sequence:

$$
0 \rightarrow \mathscr{L} \rightarrow \mathscr{O}_{C} \rightarrow \mathscr{O}_{\mathbb{P}^{1}} \rightarrow 0 .
$$

Define the arithmetic genus $g$ of a ribbon $C$ as

$$
g=1-\chi\left(\mathscr{O}_{C}\right)
$$

From the conormal sequence, we see that $C$ has genus $g$ if and only if the conormal bundle $\mathscr{L}$ on $\mathbb{P}^{1}$ is isomorphic to $\mathscr{O}_{\mathbb{P}^{1}}(-g-1)$.

There is another short exact sequence called the restricted cotangent sequence:

$$
\left.0 \rightarrow \mathscr{L} \rightarrow \Omega_{C}\right|_{\mathbb{P}^{1}} \rightarrow \Omega_{\mathbb{P}^{1}} \rightarrow 0 .
$$

This restricted cotangent sequence defines the extension class of $C$ :

$$
e_{c} \in \operatorname{Ext}_{\mathbb{P}^{1}}^{1}\left(\Omega_{\mathbb{P}^{1}}, \mathscr{L}\right) \text {. }
$$

We will say that two ribbons are isomorphic over $\mathbb{P}^{1}$ if there is an isomorphism between them that extends the identity on $\mathbb{P}^{1}$. A ribbon $C$ is split if the inclusion $\mathbb{P}^{1} \hookrightarrow C$ admits a section. Such a section is a scheme-theoretically degree two map from $C$ to $\mathbb{P}^{1}$. We also call $C$ hyperelliptic if it is split.

Received by the editors April 6, 2009 and, in revised form, January 24, 2010.

2010 Mathematics Subject Classification. Primary 14H51, 14M12, 15 A03.

(C) 2010 American Mathematical Society 
Theorem 1.1. Given any line bundle $\mathscr{L}$ on $\mathbb{P}^{1}$ and any class $e \in \operatorname{Ext}_{\mathbb{P}^{1}}^{1}\left(\Omega_{\mathbb{P}^{1}}, \mathscr{L}\right)$, there is a unique ribbon $C$ on $\mathbb{P}^{1}$ with $e_{c}=e$. If there is another class $e^{\prime} \in$ $\operatorname{Ext}_{\mathbb{P}^{1}}^{1}\left(\Omega_{\mathbb{P}^{1}}, \mathscr{L}\right)$ corresponding to a ribbon $C^{\prime}$, then $C \cong C^{\prime}$ if and only if $e=a e^{\prime}$ for some $a \in k^{*}$. A hyperelliptic ribbon corresponds to the split extension. The set of nonhyperelliptic ribbons of genus $g$, up to isomorphism over $\mathbb{P}^{1}$, is the set

$$
\mathbb{P}^{g-3}=\mathbb{P}\left(H^{0}\left(\mathscr{O}_{\mathbb{P}^{1}}(g-3)\right)\right) .
$$

Proof. The above results are essentially from [BE, Thm. 1.2, 2.1].

There is an explicit way to write down the structure of a ribbon by a gluing method; cf. [BE, Sec. 3]. Define two open sets

$$
u_{1}=\operatorname{Spec} k[s], u_{2}=\operatorname{Spec} k[t]
$$

that cover $\mathbb{P}^{1}$ via the identification $s^{-1}=t$ on $u_{1} \cap u_{2}$.

If $C$ is a genus $g$ ribbon on $\mathbb{P}^{1}$, we can write

$$
\begin{aligned}
& U_{1}:=\left.C\right|_{u_{1}} \cong \operatorname{Spec} k[s, \epsilon] / \epsilon^{2}, \\
& U_{2}:=\left.C\right|_{u_{2}} \cong \operatorname{Spec} k[t, \eta] / \eta^{2} .
\end{aligned}
$$

$C$ may be specified by a gluing isomorphism between $U_{1}$ and $U_{2}$ over $u_{1} \cap u_{2}$. The ideal sheaf $\mathscr{L} \cong \mathscr{O}_{\mathbb{P}^{1}}(-g-1)$ of $\mathbb{P}^{1}$ in $C$ is generated by $\epsilon$ on $u_{1}$ and by $\eta$ on $u_{2}$. So we can further write

$$
\begin{gathered}
\epsilon=t^{-g-1} \eta, \\
s^{-1}=t+F(t) \eta
\end{gathered}
$$

on $u_{1} \cap u_{2}$, with $F(t) \in k\left[t, t^{-1}\right]$. Conversely, any such gluing data can determine a ribbon of genus $g$ on $\mathbb{P}^{1}$.

If we change the coordinates with

$$
s^{\prime}=s+p(s) \epsilon, t=t^{\prime}+q(t) \eta
$$

on $U_{1}$ and $U_{2}$ with polynomials $p(s), q(t)$, then we get

$$
\begin{aligned}
s^{-1} & =s^{-1}-s^{-2} p(s) \epsilon \\
& =t^{\prime}+\left(F(t)-t^{-g+1} p\left(t^{-1}-F(t) \eta\right)+q(t)\right) \eta \\
& =t+F(t) \eta-(t+F(t) \eta)^{2} p\left(t^{-1}\right) t^{-g-1} \eta \\
& =t+F(t) \eta-t^{1-g} p\left(t^{-1}\right) \eta \\
& =t^{\prime}+\left(F(t)+q(t)-t^{1-g} p\left(t^{-1}\right)\right) \eta \\
& =t^{\prime}+\left(F\left(t^{\prime}\right)+q\left(t^{\prime}\right)-t^{\prime 1-g} p\left(t^{\prime-1}\right)\right) \eta .
\end{aligned}
$$

The fact that $t \eta=t^{\prime} \eta$ is used in the last step. If we multiply $s$ or $t$ by a scalar, $F$ will also be multiplied by the same scalar. Therefore, $F$ can be determined as an element of the projective space of lines in the quotient

$$
k\left[t, t^{-1}\right] /\left(k[t]+t^{-g+1} k\left[t^{-1}\right]\right) .
$$

From now on, we shall write $F$ as

$$
F=\sum_{i=1}^{g-2} F_{i} t^{-i} .
$$


$F=0$ corresponds to a hyperelliptic ribbon. This explicit expression recovers the fact in Theorem 1.1 that nonhyperelliptic ribbons of genus $g$, up to isomorphism over $\mathbb{P}^{1}$, are parameterized by the set

$$
\mathbb{P}^{g-3}=\mathbb{P}\left(H^{0}\left(\mathscr{O}_{\mathbb{P}^{1}}(g-3)\right)\right) .
$$

Now we consider line bundles. Let $L$ be a line bundle on a ribbon $C$. The degree of $L$ is defined as

$$
\operatorname{deg} L:=\chi(L)-\chi\left(\mathscr{O}_{C}\right) .
$$

Proposition 1.2. If $\left.L\right|_{\mathbb{P}^{1}} \cong \mathscr{O}_{\mathbb{P}^{1}}(n)$, then $\operatorname{deg} L=2 n$. The Picard group of $C$ is

$$
\text { Pic } C=H^{1}\left(\mathscr{O}_{\mathbb{P}^{1}}(-g-1)\right) \times \mathbb{Z} \cong k^{g} \times \mathbb{Z},
$$

where the projection to $\mathbb{Z}$ is given by the degree of the restriction $\left.L\right|_{\mathbb{P}^{1}}$.

Proof. See [BE, Props. 4.1, 4.2].

Bayer and Eisenbud remarked that one must switch to torsion-free sheaves in order to obtain the analogue of line bundles of odd degree. For simplicity, here we only consider line bundles of even degree $2 n$.

A line bundle $L$ can also be constructed by gluing. Suppose $\left.L\right|_{\mathbb{P}^{1}} \cong \mathscr{O}_{\mathbb{P}^{1}}(n)$. Using the above notation, we have

$$
\begin{aligned}
& \left.L\right|_{U_{1}}=k[s, \epsilon] e_{1}, \\
& \left.L\right|_{U_{2}}=k[t, \eta] e_{2},
\end{aligned}
$$

and

$$
e_{1}=(t+F \eta)^{n}(1+G \eta) e_{2}
$$

on $U_{1} \cap U_{2}$ for some $G \in k\left[t, t^{-1}\right]$. Conversely, any such $G$ can be used to construct a line bundle.

If we change the coordinates by

$$
e_{1}=(1+m(s) \epsilon)^{-1} e_{1}^{\prime}, e_{2}=(1+n(t) \eta) e_{2}^{\prime}
$$

on $U_{1}$ and $U_{2}$ with polynomials $m(s), n(t)$, then we get

$$
e_{1}^{\prime}=(t+F \eta)^{n}\left(1+\left(G-m\left(t^{-1}\right) t^{-g-1}+n(t)\right) \eta\right) e_{2}^{\prime} .
$$

In order to classify $L$, it suffices to specify $G$ as an element of

$$
k\left[t, t^{-1}\right] /\left(k[t]+t^{-g-1} k\left[t^{-1}\right]\right)=H^{1}\left(\mathscr{O}_{\mathbb{P}^{1}}(-g-1)\right) .
$$

This also recovers the fact in Proposition 1.2 that $H^{1}\left(\mathscr{O}_{\mathbb{P}^{1}}(-g-1)\right)$ parameterizes line bundles of fixed degree. We will also write $G$ as

$$
G=\sum_{j=1}^{g} G_{j} t^{-j}
$$

Let $L$ be a line bundle on $C$ of degree $2 n$ given by the above gluing data. We would like to find out the space of global sections of $L$. Suppose $p=p(t)$ is a polynomial of degree $\leq n$. Then $\left.p e_{2}\right|_{\mathbb{P}^{1}}$ determines an element

$$
\sigma \in H^{0}\left(\left.L\right|_{\mathbb{P}^{1}}\right)=H^{0}\left(\mathscr{O}_{\mathbb{P}^{1}}(n)\right) .
$$

Define

$$
\delta_{L}(p)=-\left(p^{\prime} F+p G\right) \in k\left[t, t^{-1}\right] /\left(k[t]+t^{n-g-1} k\left[t^{-1}\right]\right),
$$

where $p^{\prime}=\frac{\partial p}{\partial t}$. 
Theorem 1.3. The space of sections of $L$ restricted to $U_{2}=\operatorname{Spec} k[t, \eta]$ can be identified as the direct sum of the space of elements $q(t) \eta$ and the space of expressions $p(t)+p_{1}(t) \eta$, where $q$ is a polynomial of degree $\leq n-g-1, p$ is a polynomial of degree $\leq n$ satisfying $\delta_{L}(p)=0$ in (3), and $p_{1} \in k[t]$ is the polynomial part of $p^{\prime} F+p G$, i.e. $p_{1}(t) \equiv p^{\prime}(t) F(t)+p(t) G(t) \bmod t^{-1} k\left[t^{-1}\right]$.

Proof. This is exactly [BE, Thm. 4.3].

At first glance, the above way to identify $H^{0}(L)$ seems quite messy. Nevertheless, a further observation will imply an important conclusion immediately.

Corollary 1.4. Let $L$ be a line bundle of degree $2 n$ on a ribbon $C$. If $n \geq g$, then $h^{0}(L)=1-g+2 n$.

Proof. Let a section of $L$ restricted to $U_{2}$ correspond to the data $\left(q(t) \eta, p(t)+p_{1}(t)\right)$ in the above setup. When $n \geq g$, we have $k\left[t, t^{-1}\right] /\left(k[t]+t^{n-g-1} k\left[t^{-1}\right]\right) \equiv 0$. Then by its definition, $\delta_{L}$ always takes the value of 0 . So $\delta_{L}(p)=0$ does not impose any condition on $p(t)$. The only constraint on $p(t)$ and $q(t)$ is the upper bound of their degree. Here $q(t)$ has degree $\leq n-g-1$ and $p(t)$ has degree $\leq n$. In total, they yield a $(1-g+2 n)$-dimensional space for sections of $L$.

Remark 1.5. Notice that if $n \geq g$, then the degree $d$ of $L$ satisfies $d=2 n>2 g-2$. In case $C$ is a smooth curve of genus $g, h^{0}(L)=1-g+d$ holds for any line bundle $L$ on $C$ with degree $d>2 g-2$. So the above corollary can be viewed as a similarity between ribbons and smooth curves.

\section{ThE LOCUS $W_{2 n}^{r}$}

For smooth curves, the theory of special linear series can be best characterized by the Brill-Noether theory. We refer readers to ACGH, Chap. V] for bibliographical notes on this topic. Let $C$ be a curve of genus $g$. We introduce the variety $W_{d}^{r}(C)$ as

$$
W_{d}^{r}(C)=\left\{L \in \operatorname{Pic}^{d}(C) \mid h^{0}(C, L) \geq r+1\right\} .
$$

We also define the Brill-Noether number $\rho$ as

$$
\rho=g-(r+1)(g-d+r) .
$$

The basic results of the Brill-Noether theory can be summarized as follows.

Theorem 2.1. Let $C$ be a smooth curve of genus $g$. Let $d, r$ be integers such that $d \geq 1, r \geq 0$.

(Existence) If $\rho \geq 0, W_{d}^{r}(C)$ is non-empty. Furthermore, every component of $W_{d}^{r}(C)$ has dimension at least equal to $\rho$ provided $r \geq d-g$.

(Connectedness) Assume that $\rho \geq 1$. Then $W_{d}^{r}(C)$ is connected.

(Dimension) Assume that $C$ is a general curve. If $\rho<0$, then $W_{d}^{r}(C)$ is empty. If $\rho \geq 0$, then $W_{d}^{r}(C)$ is reduced and of dimension $\rho$.

We would like to investigate linear series for ribbons. The importance of such a study is three-fold. In the first place, $W_{d}^{r}(C)$ essentially carries a determinantal structure for a smooth curve $C$. In case $C$ is a ribbon, the determinantal characterization can even be made explicit. Secondly, the Brill-Noether theory for a special member in a family of curves usually reveals information for a general one. Ribbons do arise as the degeneration of smooth curves; cf. [F]. Finally, Lazarsfeld $[\mathrm{L}$. proved that a general curve contained in certain K3 surfaces satisfies the above dimension 
theorem. Correspondingly, ribbons lie on the so-called K3 carpet, i.e. a double structure on a rational normal scroll, which has the same numerical invariants as a smooth K3 surface; cf. [BE, Sec. 8]. Hence, it would be interesting to figure out some results of Brill-Noether type for ribbons.

Let $C$ be a ribbon determined by $\left[F_{1}, \ldots, F_{g-2}\right]$, the coefficients of $F$ in (1), up to a scalar. Let $L$ be a line bundle of degree $2 n$ on $C$ determined by $\left(G_{1}, \ldots, G_{g}\right)$, the coefficients of $G$ in (2). If $n \geq g$, there is no special linear system because of Corollary 1.4. Actually we only need to consider $2 n \leq g-1$, since the RiemannRoch formula also holds for ribbons; cf. [BE, Sec. 5]. From now on, assume that $2 n \leq g-1$. Define a $(g-n) \times(n+1)$ matrix $\mathcal{A}_{F}(G)$ with entries $s_{i j}=$ $G_{i+j-1}+(j-1) F_{i+j-2}$, namely,

$$
\mathcal{A}_{F}(G)=\left(\begin{array}{cccc}
G_{1} & G_{2}+F_{1} & \cdots & G_{n+1}+n F_{n} \\
G_{2} & G_{3}+F_{2} & \cdots & G_{n+2}+n F_{n+1} \\
\vdots & \vdots & \ddots & \vdots \\
G_{g-n} & G_{g-n+1}+F_{g-n} & \cdots & G_{g}
\end{array}\right) .
$$

Now we can state our main result.

Theorem 2.2. In the above setting, the locus $W_{2 n}^{r}(C)$ is isomorphic to the following affine algebraic set:

$$
W_{2 n}^{r}(C)=\left\{\left(G_{1}, \ldots, G_{g}\right) \in \mathbb{A}^{g} \mid \operatorname{rank} \mathcal{A}_{F}(G) \leq n-r\right\} .
$$

Proof. By Theorem 1.3, the space $H^{0}(L)$ of global sections of $L$ can be identified as the direct sum of two spaces:

$$
\langle q(t) \eta\rangle \oplus\left\langle p(t)+p_{1}(t) \eta\right\rangle .
$$

Since $q(t)$ is a polynomial of degree $\leq n-g-1$ and $n \leq g-2$, the first summand is the null space. For the second, $p(t)$ is a polynomial of degree $\leq n$ satisfying $\delta_{L}(p)=0$ as in (3). Then $p_{1}(t)$ is determined by $p(t)$, the polynomial part of $p^{\prime} F+p G$. Let $p(t)=\sum_{i=0}^{n} a_{i} t^{i}$. The condition $\delta_{L}(p)=0$ means

$$
p^{\prime} F+p G \in k[t]+t^{n-g-2} k\left[t^{-1}\right],
$$

which is equivalent to the following:

$$
\mathcal{A}_{F}(G) \cdot \vec{a}=0,
$$

where $\vec{a}$ is the vector $\left(a_{0}, \ldots, a_{n}\right)^{t}$ determined by the coefficients of $p(t)$. Note that $g-n \geq n+1$ by the assumption on $n$. Hence, $W_{2 n}^{r}(C)$ can be identified as the desired determinantal locus.

The following Clifford theorem for ribbons is a direct consequence of Theorem 2.2 .

Theorem 2.3. Let $C$ be a ribbon and let $L$ be a line bundle of degree $2 n$ on $C$, $1 \leq n \leq g-2$. Then $h^{0}(C, L) \leq n+1$. The equality holds if and only if $C$ is a hyperelliptic ribbon and $L$ is the pullback of $\mathscr{O}_{\mathbb{P}^{1}}(n)$ from $C_{\text {red }} \cong \mathbb{P}^{1}$ to $C$.

Proof. By the above determinantal description for $W_{2 n}^{r}(C)$, we know that $h^{0}(C, L)$ $\leq n+1$. If the equality holds, then $r=n$. We have $G_{i}=0$ and $F_{j}=0$ for all $i, j$. Thus $C$ is hyperelliptic and $L$ is isomorphic to the pullback of $\mathscr{O}_{\mathbb{P}^{1}}(n)$ from $C_{\text {red }} \cong \mathbb{P}^{1}$. 
When $C$ is a hyperelliptic ribbon, i.e. $F_{i}=0$ for all $i, \mathcal{A}_{F}(G)$ has entries $s_{i j}=G_{i+j-1}$ :

$$
\left(\begin{array}{cccc}
G_{1} & G_{2} & \cdots & G_{n+1} \\
G_{2} & G_{3} & \cdots & G_{n+2} \\
\vdots & \vdots & \ddots & \vdots \\
G_{g-n} & G_{g-n+1} & \cdots & G_{g}
\end{array}\right) .
$$

Such a matrix is called the catalecticant matrix. We cite the result [E, Prop. 4.3] as follows.

Proposition 2.4. The space of rank $\leq m$ catalecticant matrices is isomorphic to a cone over $S_{m}$, where $S_{m}$ is the union of $m$-secant $(m-1)$-planes to a rational normal curve of degree $g-1$.

This exactly describes $W_{2 n}^{r}(C)$ for a hyperelliptic ribbon.

Theorem 2.5. Let $C$ be a hyperelliptic ribbon. Then $W_{2 n}^{r}(C)$ is isomorphic to a cone over $S_{n-r}$ for $r<n$. In particular, $W_{2 n}^{r}(C)$ has dimension equal to $2 n-2 r$.

Proof. $W_{2 n}^{r}(C)$ can be identified as the space of rank $\leq n-r$ catalecticant matrices, which is isomorphic to a cone over $S_{n-r}$ by Proposition 2.4. Also, $S_{n-r}$ has dimension $2 n-2 r-1$, so $W_{2 n}^{r}(C)$ has dimension $2 n-2 r$.

We have seen that for a nonhyperelliptic ribbon, its structure can be determined by the data $\left[F_{1}, \ldots, F_{g-2}\right]$ in (1) as a point of $\mathbb{P}^{g-3}$. Note that the expected dimension of $W_{2 n}^{r}(C)$ would still be $g-(r+1)(g-2 n+r)$, which equals the Brill-Noether number $\rho$. We would like to study the actual dimension of $W_{2 n}^{r}(C)$. First, let us focus on a natural compactification of $W_{2 n}^{r}(C)$ as follows.

Define another $(g-n) \times(n+1)$ matrix $\overline{\mathcal{A}}_{F}(G)$ with entries $s_{i j}=G_{i+j-1}+$ $(j-1) F_{i+j-2} G_{0}$ :

$$
\overline{\mathcal{A}}_{F}(G)=\left(\begin{array}{cccc}
G_{1} & G_{2}+F_{1} G_{0} & \cdots & G_{n+1}+n F_{n} G_{0} \\
G_{2} & G_{3}+F_{2} G_{0} & \cdots & G_{n+2}+n F_{n+1} G_{0} \\
\vdots & \vdots & \ddots & \vdots \\
G_{g-n} & G_{g-n+1}+F_{g-n} G_{0} & \cdots & G_{g}
\end{array}\right) .
$$

Let

$$
\bar{W}_{2 n}^{r}(C)=\left\{\left[G_{0}, G_{1}, \ldots, G_{g}\right] \in \mathbb{P}^{g} \mid \operatorname{rank} \overline{\mathcal{A}}_{F}(G) \leq n-r\right\} .
$$

There is an inclusion $W_{2 n}^{r}(C) \subset \bar{W}_{2 n}^{r}(C)$ given by

$$
\left(G_{1}, \ldots, G_{g}\right) \rightarrow\left[1, G_{1}, \ldots, G_{g}\right] .
$$

The complement of $W_{2 n}^{r}(C)$ in $\bar{W}_{2 n}^{r}(C)$ is just the hyperplane section $\left\{G_{0}=0\right\} \cap$ $\bar{W}_{2 n}^{r}(C)$.

We also need to introduce generic determinantal varieties. Let $M$ be the space of $(g-n) \times(n+1)$ matrices. Denote by $M_{l}$ the locus of rank $\leq l$ matrices. Here $M_{l}$ is called the $l$-generic determinantal variety, $l \leq n+1$. Denote by $\mathbf{M}$ and $\mathbf{M}_{l}$ the projectivization of $M$ and $M_{l}$ respectively.

Proposition 2.6. $\mathbf{M}_{l}$ is an irreducible subvariety of codimension $(g-n-l)(n+1-l)$ in $\mathbf{M}$.

One can refer to ACGH, Chap. II] for a general discussion on determinantal varieties. Our next result is about the global geometry of $\bar{W}_{2 n}^{r}(C)$. 
Theorem 2.7. Let $C$ be a ribbon of genus $g$. For $r<n, \bar{W}_{2 n}^{r}(C)$ is always nonempty and has dimension equal to $2 n-2 r-1$ or $2 n-2 r$. Each irreducible component of $\bar{W}_{2 n}^{r}(C)$ has dimension at least equal to $\rho$ provided $\rho \geq 0$. Furthermore, $\bar{W}_{2 n}^{r}(C)$ is connected provided $\rho>0$.

Proof. $\bar{W}_{2 n}^{r}(C)$ is the intersection of $\mathbf{M}_{n-r}$ and a $g$-dimensional linear subspace of $\mathbf{M}$ determined by $s_{i j}=G_{i+j-1}+(j-1) F_{i+j-2} G_{0}$. Therefore, each irreducible component of $\bar{W}_{2 n}^{r}(C)$ has dimension $\geq \operatorname{dim} \mathbf{M}_{n-r}+g-\operatorname{dim} \mathbf{M}=\rho$.

When $G_{0}=0$, the matrix $\overline{\mathcal{A}}_{F}(G)$ reduces to a catalecticant matrix with entries $s_{i j}=G_{i+j-1}$. The space of rank $\leq n-r$ catalecticant matrices has dimension $2 n-2 r$ by Proposition 2.4. It implies that the hyperplane section $\left\{G_{0}=0\right\} \cap \bar{W}_{2 n}^{r}(C)$ has dimension $2 n-2 r-1$. If the top dimensional component of $\bar{W}_{2 n}^{r}(C)$ is contained in $\left\{G_{0}=0\right\}$, then $\bar{W}_{2 n}^{r}(C)$ has dimension $2 n-2 r-1$. Otherwise it has dimension $2 n-2 r$.

$\bar{W}_{2 n}^{r}(C)$ can also be regarded as the intersection of the $(n-r)$-generic determinantal variety $\mathbf{M}_{n-r}$ and a $g$-dimensional linear subspace of $\mathbf{M}$ defined by $s_{i j}=G_{i+j-1}+(j-1) F_{i+j-2} G_{0}$ for a fixed lifting $\left(F_{1}, \ldots, F_{g-2}\right)$. If $\rho>0$, the sum of the dimensions of these two spaces is greater than the dimension of $\mathbf{M}$. The connectedness of their intersection $\bar{W}_{2 n}^{r}(C)$ follows as a consequence of $\mathrm{L}, \mathrm{Ex}$. 3.3.7].

Corollary 2.8. Assume that $\rho \geq 0$. If $W_{2 n}^{r}(C)$ is non-empty for a ribbon $C$, then $W_{2 n}^{r}(C)$ has dimension at least equal to $\rho$.

Proof. $W_{2 n}^{r}(C)$ is the complement of the hyperplane section $\left\{G_{0}=0\right\} \cap \bar{W}_{2 n}^{r}(C)$ in $\bar{W}_{2 n}^{r}(C)$. By Theorem 2.7 we know that each component of $\bar{W}_{2 n}^{r}(C)$ has dimension $\geq \rho$. So does $W_{2 n}^{r}(C)$, assuming it is non-empty.

We can also let $\left(F_{1}, \ldots, F_{g-2}\right)$ vary as a point of $\mathbb{A}^{g-2}$ and define the global Brill-Noether locus $\mathcal{W}_{2 n}^{r}$ as follows:

$$
\mathcal{W}_{2 n}^{r}=\left\{\left(G_{1}, \ldots, G_{g} ; F_{1}, \ldots, F_{g-2}\right) \in \mathbb{A}^{g} \times \mathbb{A}^{g-2} \mid \operatorname{rank} \mathcal{A}_{F}(G) \leq n-r\right\} .
$$

$\mathcal{W}_{2 n}^{r}$ is the intersection of the $(n-r)$-generic determinantal variety $M_{n-r}$ and a $(2 g-2)$-dimensional linear subspace $S$ of $M$, where $S$ is determined by relations $2 s_{i j}=s_{i-1 j+1}+s_{i+1 j-1}$. Note that the expected dimension of $\mathcal{W}_{2 n}^{r}$ would be $2 g-2-(g-2 n+r)(r+1)=g-2+\rho$, which implies the following conclusion right away.

Corollary 2.9. If $\mathcal{W}_{2 n}^{r}$ has dimension equal to $g-2+\rho$, then for $\left(F_{1}, \ldots, F_{g-2}\right)$ corresponding to a general ribbon $C, W_{2 n}^{r}(C)$ has dimension at most equal to $\rho$.

In order to calculate the actual dimension of $\mathcal{W}_{2 n}^{r}$, we introduce the concept of l-generic spaces developed by Eisenbud [E, Prop.-Def. 1.1].

Definition 2.10. Let $L$ be a linear subspace of the space $M$ of $(g-n) \times(n+1)$ matrices. Here $L$ can be regarded as an associated $(g-n) \times(n+1)$ matrix of linear forms. We say that $L$ is $m$-generic for some integer $1 \leq m \leq n+1$ if after arbitrary invertible row and column operations, any $m$ of the linear forms $L_{i j}$ in $L$ are linearly independent.

We also say that $L$ meets $M_{l}$ properly if their intersection has codimension equal to $(g-n-l)(n+1-l)$ in $L$. 
Theorem 2.11. Let $L \subset M$ be an m-generic space; then $L$ meets $M_{n+1-m}$ properly.

Proof. This is part of [E, Thm. 2.1].

Note that the space of catalecticant matrices is 1-generic. One can also prove the 2-genericity for the space $S$ of matrices of type $\mathcal{A}_{F}(G)$.

Proposition 2.12. Consider $G_{1}, \ldots, G_{g} ; F_{1}, \ldots, F_{g-2}$ as independent linear forms. The $(2 g-2)$-dimensional vector space $S$ of all matrices determined by $\mathcal{A}_{F}(G)$ is 2-generic.

Proof. $\mathcal{A}_{F}(G)$ is the matrix with entries $s_{i j}=G_{i+j-1}+(j-1) F_{i+j-2}$. Suppose there were two invertible matrices $A=\left(a_{i j}\right)$ and $B=\left(b_{i j}\right)$ corresponding to invertible row and column operations such that two entries of the new matrix $A \cdot \mathcal{A}_{F}(G) \cdot B=\left(s_{i j}^{\prime}\right)$ became equal to each other. We can always assume that these two entries are $s_{11}^{\prime}$ and $s_{22}^{\prime}$. The case that they are in the same row or column would be even easier. Then the condition $s_{11}^{\prime}=s_{22}^{\prime}$ is equivalent to

$$
\sum_{i+j=k+1}\left(a_{1 i} b_{j 1}-a_{2 i} b_{j 2}\right)\left(G_{k}+(j-1) F_{k-1}\right)=0
$$

for any $k$. Namely,

$$
\sum_{i+j=k+1}\left(a_{1 i} b_{j 1}-a_{2 i} b_{j 2}\right)=0 \text { and } \sum_{i+j=k+1}\left(a_{1 i} b_{j 1}-a_{2 i} b_{j 2}\right)(j-1)=0
$$

since $G_{i}$ and $F_{j}$ can vary independently.

Define four polynomials as follows:

$$
A_{k}(x)=\sum_{i} a_{k i} x^{i} \text { and } B_{k}(x)=\sum_{j} b_{k j} x^{j} \text { for } k=1,2 .
$$

We can deduce from the above two equalities that

$$
A_{1}(x) B_{1}(x)=A_{2}(x) B_{2}(x) \text { and } A_{1}(x) B_{1}^{\prime}(x)=A_{2}(x) B_{2}^{\prime}(x) .
$$

Since the matrices $A$ and $B$ are invertible, by these two relations we can get

$$
B_{1}(x) B_{2}^{\prime}(x)=B_{2}(x) B_{1}^{\prime}(x)
$$

which would imply that $\left(B_{1}(x) / B_{2}(x)\right)^{\prime}=0$. Then $B_{1}(x) / B_{2}(x)$ would be a constant, which contradicts the assumption that the matrix $B$ is invertible.

Corollary 2.13. For $r=1, \mathcal{W}_{2 n}^{1}$ has dimension $4 n-4$ and $W_{2 n}^{1}(C)$ has dimension at most equal to $\rho=4 n-g-2$ for a general ribbon $C$, provided $\rho \geq 0$.

Proof. Since the space of matrices $\overline{\mathcal{A}}_{F}(G)$ is 2-generic, it intersects $M_{n-1}$ properly. So the intersection $\mathcal{W}_{2 n}^{1}$ has dimension equal to $g-2+\rho=4 n-4$ by Theorem 2.11. Then by Corollary 2.9. $W_{2 n}^{1}(C)$ has dimension at most equal to $\rho=4 n-2-g$.

Based on Corollaries 2.8 and 2.13, we obtain the following conclusion.

Corollary 2.14. For $r=1$, if $W_{2 n}^{1}(C)$ is non-empty for a general ribbon $C$, then $W_{2 n}^{1}(C)$ has dimension equal to $\rho=4 n-2-g$ provided $\rho \geq 0$.

It would be interesting to pin down the following question in general. 
Question 2.15. For $\rho \geq 0$, is the dimension of $\mathcal{W}_{2 n}^{r}$ equal to the expected dimension $g-2+\rho$ ? For a general ribbon $C$, is the locus $W_{2 n}^{r}(C)$ non-empty and does it have dimension equal to $\rho$ provided $\rho \geq 0$ ?

By the determinantal descriptions for $\mathcal{W}_{2 n}^{r}$ and $W_{2 n}^{r}(C)$, using Macaulay one can check that the above question does have a positive answer when the genus of $C$ is small.

\section{ACKNOWLEDGEMENTS}

I am grateful to David Eisenbud for enlightening discussions on determinantal varieties, to Gregory Smith for his help on Macaulay, to Nicolae Manolache for pointing out a splitting of terminology in the literature, and to the referee for carefully reading this paper. Part of the work was finished during the Algebraic Geometry MSRI 2009 Program. I want to thank MSRI for providing a wonderful working environment. Finally, I would like to thank Yongnam Lee and Jerzy Weyman for inviting me to the AMS-KMS meeting at Seoul, December 2009, where this work was reported.

\section{REFERENCES}

[ACGH] E. Arbarello, M. Cornalba, P. A. Griffiths and J. Harris, Geometry of algebraic curves, Grundlehren der Mathematischen Wissenschaften, 267, Springer-Verlag, New York, 1985. MR770932 (86h:14019)

[BE] D. Bayer and D. Eisenbud, Ribbons and their canonical embeddings, Trans. Amer. Math. Soc. 347 (1995), no. 3, 719-756. MR.1273472 (95g:14032)

[E] D. Eisenbud, Linear sections of determinantal varieties, Amer. J. Math. 110 (1988), no. 3, 541-575. MR944327 (89h:14041)

[F] L.-Y. Fong, Rational ribbons and deformation of hyperelliptic curves, J. Algebraic Geom. 2 (1993), no. 2, 295-307. MR.1203687 (94c:14020)

[L] R. Lazarsfeld, Brill-Noether-Petri without degenerations, J. Differential Geom. 23 (1986), no. 3, 299-307. MR852158 (88b:14019)

Department of Mathematics, Statistics, and Computer Science, University of Illinois at Chichag, Chicago, Illinois 60607

E-mail address: dwchen@math.uic.edu 